\title{
Lyapunov inequality for a boundary value problem involving conformable derivative
}

\author{
Rabah Khaldi, Assia Guezane-Lakoud \\ Laboratory of Advanced Materials, Departement of Mathematics, Badji Mokhtar-Annaba University, P.O. Box 12, 23000 Annaba, \\ 'Algeria
}

Received: 3 Apr. 2017, Revised: 9 May 2017, Accepted: 15 May 2017

'Published online: .

Abstract: We consider a boundary value problem involving conformable derivative of order $\alpha, 1<\alpha<2$ and Dirichlet conditions. To prove the existence of solutions, we apply the method of upper and lower solutions together with Schauder's fixed-point theorem. Futhermore, we give the Lyapunov inequality for the corresponding problem.

Keywords: Boundary value problem, Lyapunov inequality, Conformable derivative, Upper and lower solutions method, Existence of solution.

2010 Mathematics Subject Classification. Primary 34B15, 34A08; Secondary 26A33, 34A12.

\section{Introduction}

Recently, an interesting derivative called conformable derivative that is based on a limit form as in the classical derivative 'was introduced by Khalil et al. in [20]. Later, this new local derivative is getting more attention and is improved by Abdeljawad in [1]. The importance of the conformable derivative is that it has similar properties than the classical one. Nevertheless, this conformable derivative doesn't satisfy the index law [18,24] and the zero order derivative property i.e. the zero order derivative of a differentiable function does not return to the function itself.

Following this new conformable derivative, several papers have been presented, in particular some studies about boundary value problems for conformable differential equations have been the subject of some papers [3-8,18,24,26]. Furthermore, in [6], Batarfi et al. studied a conformable differential equation of order $\alpha \in(1,2]$, with three point boundary 'conditions and proved the existence and uniqueness of solution by using fixed point theorems. In [7], Bayour et al. solved an initial conformable differential value problem for $\alpha \in(0,1)$ by the help of the tube solution method which is a generalization of the lower and upper solutions method.

In this work, we analyze the existence of solutions for the following boundary value problem (P)

$$
\begin{gathered}
T_{\alpha}^{a} u(t)+f(t, u(t))=0, a<t<b, \\
u(a)=u(b)=0
\end{gathered}
$$

where $1<\alpha<2, T_{\alpha}^{a}$ denotes the conformable derivative of order $\alpha, u$ is the unknown function and $f:[a, b] \times \mathbb{R} \rightarrow \mathbb{R}$ is a given function. For this purpose, we use the method of upper and lower solutions together with Schauder's fixed-point theorem. The method of lower and upper solutions is a powerful tool in the investigation of the existence of solutions and has been used in several papers, we refer to $[10,13,15,19]$.

In the case $f(t, u(t))=q(t) u(t)$, we prove a new Lyapunov inequality that coincide with the classical one when $\alpha=2$.

The classical Lyapunov inequality states that if $q:[a, b] \rightarrow R$ is a real and continuous function, then a necessary condition for the boundary value problem 


$$
\begin{gathered}
-u^{\prime \prime}(t)=q(t) u(t), a<t<b \\
u(a)=u(b)=0
\end{gathered}
$$

to have nontrivial solutions is that

$$
\int_{a}^{b}|q(t)| d t \geq \frac{4}{b-a}
$$

see [21]. An equivalent version of the Lyapunov inequality (1.3) was proved by Borg see [8].

$$
\int_{a}^{b} \frac{\left|u^{\prime \prime}(t)\right|}{u(t)} d t \geq \frac{4}{b-a}
$$

under the condition $u(t)>0$ for $t \in(a, b)$.

Many authors have extended the Lyapunov inequality by considering a fractional derivative or a sequential of fractional derivatives instead of the second derivative in equation (1.1), see [2,9,11-12,14,16-17,21-23,25]. In particular, we cite the paper of Ferreira [12], where he gave the corresponding Lyapunov type inequalities for both Caputo sequential fractional differential equation and Riemann-Liouville sequential fractional differential equation subject to Dirichlet boundary conditions. In [2], Agarwal et al. obtained Lyapunov type inequalities for mixed nonlinear Riemann-Liouville fractional differential equations with a forcing term and Dirichlet boundary conditions. Recently, Guezane-Lakoud et al. [14], considered a mixed left Riemann-Liouville and right Caputo differential equation subject to natural conditions and obtained a new Lyapunov type inequality.

This paper is organized as follows. In Section 2, we present the main concepts of the conformable derivatives, we give some useful properties and we prove a property on the extremum of a function for a conformable derivative. In Section 3 , we prove existence of solution to problem (P) by using the method of upper and lower solutions together with Schauder's fixed-point theorem. In Section 4, we prove a Lyapunov inequality for problem (P) in the case $f(t, u(t))=q(t) u(t)$.

As far as we know, this work will be the first one that gives the Lyapunov inequality for conformable differential equations.

\section{Preliminaries}

We recall some essential definitions on conformable derivatives that can be found in $[1,20]$.

Let $n<\alpha<n+1$, and set $\beta=\alpha-n$, for a function $g:[a, \infty) \rightarrow \mathbb{R}$, we denote by

$$
I_{\alpha}^{a} g(t)=\int_{a}^{t}(s-a)^{\alpha-1} g(s) d s, n=0,
$$

and

$$
I_{\alpha}^{a} g(t)=\frac{1}{n !} \int_{a}^{t}(t-s)^{n} g(s) d \beta(s, a)=\frac{1}{n !} \int_{a}^{t}(t-s)^{n}(s-a)^{\beta-1} g(s) d s, n \geq 1 .
$$

Remark. Notice that, since $0<\beta<1$, then $I_{\alpha}^{a} g(t)$ is the Lebesgue-stieltjes integral of the function $(t-s)^{n} g(s)$ on $[a, t]$ and $d \beta(s, a)=(s-a)^{\beta-1} d s$ is an absolutely continuous measure with respect to the Lebesgue measure on the real line, generated by the absolutely continuous function $(t-a)^{\beta}$ and the weight function $(s-a)^{\beta-1} \in L_{1}[a, b]$ is its RadonNikodym derivative according to the Lebesgue measure.

The conformable derivative of order $0<\alpha<1$, of a function $g:[a, \infty) \rightarrow \mathbb{R}$ is defined by

$$
T_{\alpha}^{a} g(t)=\lim _{\varepsilon \rightarrow 0} \frac{g\left(t+\varepsilon(t-a)^{1-\alpha}\right)-g(t)}{\varepsilon}, t>a .
$$

If $T_{\alpha}^{a} g(t)$ exists on $(a, b), b>a$ and $\lim _{t \rightarrow a^{+}} T_{\alpha}^{a} g(t)$ exists, then we define $T_{\alpha}^{a} g(a)=\lim _{t \rightarrow a^{+}} T_{\alpha}^{a} g(t)$.

The conformable derivative of order $n<\alpha<n+1$ of a function $g:[a, \infty) \rightarrow \mathbb{R}$, when $g^{(n)}$ exists, is defined by

$$
T_{\alpha}^{a} g(t)=T_{\beta}^{a} g^{(n)}(t),
$$

where $\beta=\alpha-n \in(0,1)$. 
For the properties of the conformable derivative, we mention the following:

Let $n<\alpha<n+1$ and $g$ be an $(n+1)$-differentiable at $t>a$, then we have

$$
T_{\alpha}^{a} g(t)=(t-a)^{n+1-\alpha} g^{(n+1)}(t)
$$

and

$$
I_{\alpha}^{a} T_{\alpha}^{a} g(t)=g(t)-\sum_{k=0}^{n} \frac{g^{(k)}(a)(t-a)^{k}}{k !} .
$$

Remark. -For $0<\alpha<1$, using (2.1) it follows that, if a function $g$ is differentiable at $t>a$, then one has

$$
\lim _{\alpha \rightarrow 1} T_{\alpha}^{a} g(t)=g^{\prime}(t)
$$

and

$$
\lim _{\alpha \rightarrow 0} T_{\alpha}^{a} g(t)=(t-a) g^{\prime}(t),
$$

i.e. the zero order derivative of a differentiable function does not return to the function itself.

-Let $n<\alpha<n+1$, if $g$ is $(n+1)$-differentiable on $(a, b), b>a$ and $\lim _{t \rightarrow a^{+}} g^{(n+1)}$ exists, then from $(2.1)$, we get $T_{\alpha}^{a} g(a)=$ $\lim _{t \rightarrow a^{+}} T_{\alpha}^{a} g(t)=0$.

-Let $n<\alpha<n+1$, if $g$ is $(n+1)$-differentiable at $t>a$, then we can show that $T_{\alpha}^{a} g(t)=T_{\alpha-k}^{a} g^{(k)}(t)$ for all positive integer $k<\alpha$.

Similarly to the classical case, we give a property on the extremum of a function that has a conformable derivative:

Proposition 1. Let $1<\alpha<2$, if a function $g \in C^{1}[a, b]$ attains a global maximum (respectively minimum) at some point $\xi \in(a, b)$, then $T_{\alpha}^{a} g(\xi) \leq 0$ (respectively $T_{\alpha}^{a} g(\xi) \geq 0$ ).

Proof. The result follows from the fact that

$$
T_{\alpha}^{a} g(\xi)=T_{\alpha-1}^{a} g^{\prime}(\xi)=\lim _{\varepsilon \rightarrow 0} \frac{g^{\prime}\left(\xi+\varepsilon(\xi-a)^{2-\alpha}\right)}{\varepsilon} .
$$

\section{Existence of solutions}

Let $A C^{2}[a, b]=\left\{u \in C^{1}[a, b], u^{\prime} \in A C[a, b]\right\}$, where $A C[a, b]$ is the space of absolutely continuous functions on $[a, b]$. Denote $L^{1}([a, b], \rho(s) d s)$ the Banach space of Lebesgue integrable functions on $[a, b]$ with respect to the positive weight function $\rho(s)=(s-a)^{\alpha-2} \in L^{1}[a, b], 1<\alpha<2$.

To prove the existence of solutions for problem $(\mathrm{P})$, we use the lower and upper solutions method, we need the following definition of lower and upper solutions for problem $(\mathrm{P})$.

Definition 1. The functions $\underline{\sigma}, \bar{\sigma} \in A C^{2}[a, b]$ are called lower and upper solutions of problem $(P)$ respectively, if

a) $T_{\alpha}^{a} \underline{\sigma}(t)+f(t, \underline{\sigma}(t)) \geq 0$, for all $t \in[a, b]$,

$\underline{\sigma}(a) \leq 0, \underline{\sigma}(b) \leq 0$.

b) $T_{\alpha}^{a} \overline{\bar{\sigma}}(t)+f(t, \bar{\sigma}(t)) \leq 0$, for all $t \in[a, b]$

$\bar{\sigma}(a) \geq 0, \bar{\sigma}(b) \geq 0$.

Next, we solve the following linear boundary value problem.

Lemma 1. Assume that $y \in C[a, b]$, then the following linear boundary value problem

$$
\begin{gathered}
T_{\alpha}^{a} u(t)+y(t)=0, a<t<b, \\
u(a)=u(b)=0,
\end{gathered}
$$

has a unique solution given by

$$
u(t)=\int_{a}^{b} G(t, s) y(s) \rho(s) d s
$$

where

$$
G(t, s)=\frac{1}{(b-a)}\left\{\begin{array}{c}
-(b-a)(t-s)+(b-s)(t-a), a \leq s \leq t \leq b \\
(b-s)(t-a), a \leq t<s \leq b .
\end{array}\right.
$$


Proof. Applying the integral operator $I_{\alpha}^{a}$, to both sides of the differential equation (3.1), we get

$$
I_{\alpha}^{a} T_{\alpha}^{a} u(t)+I_{\alpha}^{a} y(t)=0
$$

hence

$$
u(t)-u(a)-c(t-a)+I_{\alpha}^{a} y(t)=0
$$

Since $u(a)=0$, then

$$
u(t)=-I_{\alpha}^{a} y(t)+c(t-a)
$$

From $u(b)=0$, we get

$$
c=\left.\frac{1}{(b-a)} I_{\alpha}^{a} y(t)\right|_{t=b}
$$

Substituting $c$ by its value in (3.4), it yields

$$
\begin{aligned}
u(t) & =-I_{\alpha}^{a} y(t)+\left.\frac{(t-a)}{(b-a)} I_{\alpha}^{a} y(t)\right|_{t=b} \\
& =-\int_{a}^{t}(t-s)(s-a)^{\alpha-2} y(s) d s+\frac{(t-a)}{(b-a)} \int_{a}^{b}(b-s)(s-a)^{\alpha-2} y(s) d s \\
& =\int_{a}^{b} G(t, s) y(s) \rho(s) d s,
\end{aligned}
$$

where the Green function $G$ is given in (3.3).

Lemma 2. The Green function $G$ is nonnegative, continuous and satisfies

$$
0 \leq G(t, s) \leq b-a, \forall s, t \in[a, b] .
$$

Now we give the main result on the existence of solutions for the nonlinear problem $(\mathrm{P})$.

Theorem 1. Let $\underline{\sigma}$ and $\bar{\sigma}$ be the lower and upper solutions of $(P)$ such that $\underline{\sigma} \leq \bar{\sigma}$, define $E=\{(t, x) \in[a, b] \times \mathbb{R}, \underline{\sigma}(t) \leq$ $x \leq \bar{\sigma}(t)\}$ and assume that $f(t, x)$ is continuous on $E$. Then the problem $(P)$ has at least one solution $u \in A C^{2}([a, b])$ such that

$$
\underline{\sigma}(t) \leq u(t) \leq \bar{\sigma}(t), a<t<b .
$$

Proof. Define the modified problem

$$
(M P)\left\{\begin{array}{c}
T_{\alpha}^{a} u(t)+F(t, u(t))=0, a<t<b \\
u(a)=u(b)=0
\end{array}\right.
$$

where

$$
F(t, x)=\left\{\begin{array}{lr}
f(t, \bar{\sigma}(t))+\frac{\bar{\sigma}(t)-x}{x-\bar{\sigma}(t)+1}, & \text { for } x>\bar{\sigma}(t), \\
f(t, x), & \text { for } \underline{\sigma}(t) \leq x \leq \bar{\sigma}(t), \\
f(t, \underline{\sigma}(t))+\frac{\underline{\sigma}(t)-x}{\underline{\sigma}(t)-x+1}, & \text { for } x<\underline{\sigma}(t) .
\end{array}\right.
$$

The function $F(t, x)$ is called a modification of $f(t, x)$ associated with the coupled of lower and upper solutions $\underline{\sigma}$ and $\bar{\sigma}$. It follows from the definition of $F$ that $F(t, x)$ is continuous and $|F(t, x)| \leq M$ on $[a, b] \times \mathbb{R}$, with $M=M_{0}+1$ where

$$
M_{0}=\max \{|f(t, x)|,(t, x) \in E\} .
$$

Define the operator $A$ on $X=C[a, b]$, by

$$
A u(t)=\int_{a}^{b} G(t, s)(s-a)^{\alpha-2} F(s, u(s)) d s, a \leq t \leq b .
$$

Set $\Omega=\left\{u \in C[a, b],|u(t)| \leq M \frac{(b-a)^{\alpha+1}}{\alpha-1}, a \leq t \leq b\right\}$. We will show that $A(\Omega)$ is uniformly bounded. Let $u \in \Omega$, then, using (3.5), we get

$$
|A u(t)| \leq \int_{a}^{b} G(t, s)(s-a)^{\alpha-2}|F(s, u(s))| d s \leq M \frac{(b-a)^{\alpha}}{\alpha-1},
$$


consequently $A(\Omega)$ is uniformly bounded and $A(\Omega) \subset \Omega$.

Now we prove that $A(\Omega)$ is equicontinuous. For $a \leq t_{1}<t_{2} \leq b$, we have

$$
\begin{aligned}
& \left|A u\left(t_{1}\right)-A u\left(t_{2}\right)\right| \\
\leq & \left(t_{2}-t_{1}\right) M \int_{a}^{t_{1}}(s-a)^{\alpha-2} d s+M \int_{t_{1}}^{t_{2}}\left(t_{2}-s\right)(s-a)^{\alpha-2} d s \\
& +\frac{\left(t_{2}-t_{1}\right) M}{(b-a)} \int_{a}^{b}(b-s)(s-a)^{\alpha-2} d s \\
\leq & \frac{M}{\alpha-1}\left[\left(t_{2}-t_{1}\right)\left(\left(t_{1}-a\right)^{\alpha-1}+(b-a)^{\alpha-2}\right)+t_{2}\left(t_{2}-t_{1}\right)^{\alpha-1}\right] \rightarrow 0,
\end{aligned}
$$

when $t_{1} \rightarrow t_{2}$. Hence, $A(\Omega)$ is equicontinuous. Thanks to Arzela-Ascoli's theorem we get that $A$ is completely continuous. Moreover, by Schauder fixed point theorem we conclude that $A$ has a fixed point $u \in \Omega$ which is a solution of the modified problem (MP).

Localization of solution. Let us prove that if $u$ is a solution of the modified problem (MP), it satisfies

$$
\underline{\sigma}(t) \leq u(t) \leq \bar{\sigma}(t) .
$$

Set $w=u-\bar{\sigma}$. Assuming the contrary, so there exists $t_{0} \in[a, b]$ such that

$$
\max _{t \in[a, b]} w(t)=w\left(t_{0}\right)>0
$$

therefore, we have some cases to consider such as the following:

Case 1: If $t_{0} \in(a, b)$, then from Proposition 1 it yields, $T_{\alpha}^{a} w\left(t_{0}\right) \leq 0$. Using the fact that $\bar{\sigma}$ is an upper solution for problem $(\mathrm{P})$, we get

$$
\begin{aligned}
T_{\alpha}^{a} w\left(t_{0}\right) & =T_{\alpha}^{a} u\left(t_{0}\right)-T_{\alpha}^{a} \overline{\bar{\sigma}}\left(t_{0}\right) \\
& =-f\left(t, \bar{\sigma}\left(t_{0}\right)\right)-\frac{\bar{\sigma}\left(t_{0}\right)-u\left(t_{0}\right)}{u\left(t_{0}\right)-\bar{\sigma}\left(t_{0}\right)+1}-T_{\alpha}^{a} \bar{\sigma}\left(t_{0}\right)>0
\end{aligned}
$$

that leads to a contradiction, thus the maximum of $w$ is not achieved at the point $t_{0} \in(a, b)$.

Case 2: If $t_{0}=a$, we obtain

$$
w(a)=u(a)-\bar{\sigma}(a)>0 .
$$

On the other hand, since $u$ is solution, then $u(a)=0$ and consequently $\bar{\sigma}(a)<0$, which contradicts the fact that $\bar{\sigma}$ is an upper solution of problem $(\mathrm{P})$.

Case 3: If $t_{0}=b$, we obtain a contradiction as in the second case.

Applying similar reasoning, we prove that $\underline{\sigma}(t) \leq u(t), \forall t \in[a, b]$. Finally from (3.6) we conclude that $u$ is a solution of problem (P). The proof is completed.

\section{Lyapunov inequality}

Let $f(t, u(t))=q(t) u(t)$, then problem $(\mathrm{P})$ becomes

$$
\begin{gathered}
T_{\alpha}^{a} u(t)+q(t) u(t)=0, a<t<b, \\
u(a)=u(b)=0,
\end{gathered}
$$

that we denote by (P1). Now we are ready to give the Lyapunov inequality for problem (P1).

Theorem 2. Let $q \in C([a, b])$. If the boundary value problem $(P 1)$ has a solution $u \in A C^{2}([a, b])$ such that $u(t) \neq 0$ a.e. on $(a, b)$, then

$$
\int_{a}^{b}|q(s)| \rho(s) d s \geq \frac{4}{b-a} .
$$


Proof. Let $u \in A C^{2}([a, b])$ be a solution of problem (P1) such that $u(t) \neq 0$ a.e. on $(a, b)$, then from equation (4.1), we can write

$$
|q(t)|=\left|\frac{T_{\alpha}^{a} u(t)}{u(t)}\right|,
$$

a.e. on $(a, b)$. Applying the integral operator $I_{\alpha-1}^{a}$ to both sides of the differential equation (4.3) and following the same ideas as in [8], we get for all $a<c<d<b$

$$
\begin{aligned}
\left.\left(I_{\alpha-1}^{a}|q|\right)(t)\right|_{t=b} & =\int_{a}^{b}(s-a)^{\alpha-2}|q(s)| d s \\
& =\int_{a}^{b}(s-a)^{\alpha-2}\left|\frac{T_{\alpha}^{a} u(s)}{u(s)}\right| d s \\
& \geq(\|u\|)^{-1} \int_{a}^{b}(s-a)^{\alpha-2}\left|(s-a)^{2-\alpha} u^{\prime \prime}(s)\right| d s \\
& \geq(\|u\|)^{-1} \int_{c}^{d}\left|u^{\prime \prime}(s)\right| d s .
\end{aligned}
$$

Since the function $u^{\prime}$ is absolutely continuous on $[a, b]$, it yields

$$
\left.\left(I_{\alpha-1}^{a}|q|\right)(t)\right|_{t=b} \geq(\|u\|)^{-1}\left|u^{\prime}(d)-u^{\prime}(c)\right|
$$

where $\|u\|=\max _{t \in[a, b]}|u(t)|$. Let $\|u\|=u(\xi)$ then the Mean value theorem implies there exist $a<c<\xi$ and $\xi<d<b$ such that

$$
\begin{aligned}
\left.\left(I_{\alpha-1}^{a}|q|\right)(t)\right|_{t=b} & \geq(\|u\|)^{-1}\left|\frac{u(b)-u(\xi)}{b-\xi}-\frac{u(\xi)-u(a)}{\xi-a}\right| \\
& =\frac{1}{b-\xi}+\frac{1}{\xi-a} .
\end{aligned}
$$

Finally thanks to the harmonic mean inequality, we get (4.2).

Remark. Note that if $\alpha \rightarrow 2$, then we get the classical Lyapunov inequality (1.3).

\section{Acknowledgement}

The authors are grateful to the anonymous referees for their valuable comments and specially grateful to The Editor-inChief Prof. Dumitru Baleanu, for his comments and suggestions that improved this paper.

\section{References}

[1] T. Abdeljawad, On conformable fractional calculus, J. Comput. Appl. Math. 279 (2015) 57-66.

[2] Ravi P. Agarwal, A. Ozbekler, Lyapunov type inequalities for mixed nonlinear Riemann-Liouville fractional differential equations with a forcing term, Journal of Computational and Applied Mathematics, (Article in press).

[3] A. Atangana, D. Baleanu, and A. Alsaedi, New properties of conformable derivative, Open Math. 13 (2015), 1-10.

[4] Atangana, A.; Baleanu, D. New Fractional Derivatives with Nonlocal and Non-Singular Kernel: Theory and Application to Heat Transfer Model. Therm. Sci. 2016, 20, 763-769.

[5] D. Baleanu, K. Diethelm, E. Scalas, J.J. Trujillo, Fractional calculus models and numerical methods, Series on Complexity, Nonlinearity and Chaos, World Scientific, Boston, 2012

[6] H. Batarfi, Jorge Losada, Juan J. Nieto and W. Shammakh, Three-Point Boundary Value Problems for Conformable Fractional Differential Equations, Journal of Function Spaces, Volume 2015 (2015), Article ID 706383, 6 pages.

[7] B. Bayour and D. F. M. Torres, Existence of solution to a local fractional nonlinear differential equation, J. Comput. Appl. Math. 312 (2017), 127-133.

[8] G. Borg, On a Liapounoff criterion of stability, Amer. J. Math. 11 (1949), 67-70.

[9] A. Chidouh, D. F. M. Torres, A generalized Lyapunov's inequality for a fractional boundary value problem, J. Comput. Appl. Math, 312 (2017), 192-197.

[10] C. De Coster and P. Habets, Two-point boundary value problems: lower and upper solutions, Mathematics in Science and Engineering, 205, Elsevier B. V., Amsterdam, 2006. 
[11] R. A. C. Ferreira, A Lyapunov-type inequality for a fractional boundary value problem, Fract. Calc. Appl. Anal. 16 (2013), no. 4, 978-984.

[12] R. A. C. Ferreira, Lyapunov-type inequalities for some sequential fractional boundary value problems, Advances in Dynamical Systems and Applications, 11, 33-43 (2016).

[13] D. Franco, J. J. Nieto and D. O'Regan, Upper and lower solutions for first order problems with nonlinear boundary conditions, Extracta Math. 18 (2), 153-160 (2003).

[14] A. Guezane-Lakoud, R. Khaldi and D. F. M. Torres, Lyapunov-type inequality for a fractional boundary value problem with natural conditions, SeMA Journal, in press. DOI: 10.1007/s40324-017-0124-2

[15] A. Guezane-Lakoud, R. Khaldi, and D. F. M. Torres, On a fractional oscillator equation with natural boundary conditions, Prog. Frac. Diff. Appl.(Forthcoming), https://arxiv.org/pdf/1701.08962.

[16] M. Jleli and B. Samet, Lyapunov-type inequalities for fractional boundary value problems,Electronic Journal of Differential Equations, Vol. 2015 (2015), No. 88, 1-11

[17] M. Jleli, L. Ragoub and B. Samet, A Lyapunov-type Inequality for a fractional differential equation under a Robin boundary condition, J. Funct. Spaces, 2015, Art. ID 468536, 5 pp.

[18] Udita N. Katugampola, Correction to "What is a fractional derivative?" by Ortigueira and Machado [Journal of Computational Physics, Volume 293, 15 July 2015, Pages 4-13. Special issue on fractional PDEs]. J. Comput. Phys. 321 (2016), 1255-1257.

[19] R. Khaldi and A. Guezane-Lakoud, Upper and lower solutions method for higher order boundary value problems, Progr. Fract. Differ. Appl. 3 (1), 53-57 (2017).

[20] R. Khalil, M. Al Horani, A. Yousef, and M. Sababheh, A new definition of fractional derivative, Journal of Computational and Applied Mathematics, vol. 264, pp. 65-70, 2014.

[21] A. M. Lyapunov, Problème général de la stabilité du mouvement, (French Translation of a Russian paper dated 1893), Ann. Fac. Sci. Univ. Toulouse 2 (1907), 27-247, Reprinted as Ann. Math. Studies, No, 17, Princeton, 1947.

[22] Constantin P. Niculescu, A new look at the Lyapunov inequality. Ann. Acad. Rom. Sci. Ser. Math. Appl. 3 (2011), no. 1, $207-217$.

[23] D. O'Regan and B. Samet, Lyapunov-type inequalities for a class of fractional differential equations, J. Inequal. Appl. 2015, 2015:247.

[24] anuel .D. Ortigueira, J.A. Tenreiro Machado, What is a fractional derivative? J. Comput. Phys. 293 (2015), 4-13

[25] J. Rong and C. Bai, Lyapunov-type inequality for a fractional differential equation with fractional boundary conditions, Adv. Difference Equ. 2015, 2015:82, 10 pp.

[26] XJ. Yang, D. Baleanu, HM. Srivastava, Local fractional integral transforms and their applications. San Diego (CA): Academic Press; 2016 
童NSD

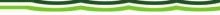

